\title{
Antisymmetric Tensor Gauge Theory
}

\author{
G.Bandelloni \\ Istituto di Fisica della Facoltá di Ingegneria - Università di Genova \\ Istituto Nazionale di Fisica Nucleare - sez. di Genova \\ Via Dodecaneso, 33 - 16146 Genova (Italy)
}

and

\begin{abstract}
A.Blasi
Dipartimento di Fisica - Università di Genova

Istituto Nazionale di Fisica Nucleare - sez. di Genova

Via Dodecaneso, 33 - 16146 Genova (Italy)
\end{abstract}

\begin{abstract}
We analyze to all perturbative orders the properties of two possible quantum extensions of classically on-shell equivalent antisymmetric tensor gauge models in four dimensions. The first case, related to the soft breaking of a topological theory wants a gauge field of canonical dimension one. The other possibility, which assigns canonical dimension two to the gauge field, leads to the $\sigma$ model interpretation of the theory. In both instances we find that the models are anomaly free.
\end{abstract}

GEF-Th-10/93

March 1993 


\section{Introduction}

The antisymmetric tensor field models have been introduced several years ago [1]; at the time the interest was focused on their dynamical relation to four dimensional $\sigma$ models

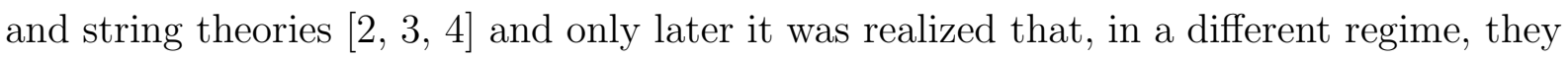
describe a topological theory which can be viewed as the natural generalization of the three-dimensional Chern-Simons model [5]. These different aspects can be illustrated, at the classical level, by the action

$$
I=-\frac{1}{2} \int d^{4} x \varepsilon^{\mu \nu \rho \sigma} F_{\mu \nu}^{a} B_{\rho \sigma}^{a}-\frac{k}{2} \int d^{4} x A_{\mu}^{a} A^{a \mu}-\frac{l}{4} \int d^{4} x F_{\mu \nu}^{a} F^{a \mu \nu}
$$

where

$$
F_{\mu \nu}^{a}=\partial_{\mu} A_{\nu}^{a}-\partial_{\nu} A_{\mu}^{a}+f^{a b c} A_{\mu}^{b} A_{\nu}^{c}
$$

and $B_{\rho \sigma}^{a}=-B_{\sigma \rho}^{a}$ is the antisymmetric tensor field.

$I$ is invariant under the B.R.S.gauge transformations

$$
\begin{aligned}
\delta A_{\mu}^{a} & =0 \\
\delta B_{\mu \nu}^{a} & =\left(D_{\mu} c_{\nu}-D_{\nu} c_{\mu}\right)^{a}
\end{aligned}
$$

with

$$
D_{\mu}^{a b}=\partial_{\mu} \delta^{a b}+f^{a c b} A_{\mu}^{c}
$$

and yields the equations of motion

$$
\frac{\delta I}{\delta B_{\mu \nu}^{a}}=-\frac{1}{2} \varepsilon^{\mu \nu \rho \sigma} F_{\mu \nu}^{a}=0
$$




$$
\frac{\delta I}{\delta A_{\mu}^{a}}=\varepsilon^{\mu \nu \rho \sigma}\left(D_{\nu} B_{\rho \sigma}\right)^{a}-k A^{a \mu}+l\left(D_{\nu} F^{\mu \nu}\right)^{a}=0
$$

which imply

$$
\begin{aligned}
A_{\mu}^{a} & =\left(U^{-1} \partial_{\mu} U\right)^{a} \\
k\left(D_{\mu} A^{\mu}\right)^{a} & =0
\end{aligned}
$$

In a suitable parametrization by means of scalar fields for the $U$ matrix, (6) yields the characteristic $\sigma$ model constraint. Proceeding in this direction we are led to attribute to the gauge field a canonical dimension equal to two [4] and therefore the $l$ coupling should be set to zero. Within this choice the symmetry (3) and the power counting are sufficient to identify the classical action.

A different interpretation is provided by the topological regime $k=l=0$ where the classical action does not contain the metric tensor. Here the switching on of the $k$ coupling can be seen as the soft breaking Đof an otherwise topological theory if the gauge field is assigned canonical dimension equal to one. In this case the symmetry (3) offers no protection against the appearance of higher dimensionality $A_{\mu}^{a}$ couplings and therefore we enlarge the invariance to include a gauge transformation for $A_{\mu}^{a}$ and consider the model for nonvanishing $l$.

The two approaches which classically describe on-shell identical models, are expected to generate different quantum extensions. The interest in considering them is twofold: first we shall provide a complete characterization of these extensions by analyzing the cohomology spaces of the corresponding nilpotent B.R.S. operators. In a perturbative framework and for both cases, we exclude the presence of anomalies, a result which was only known for the topological BF model [7] Second, for nonvanishing $k$ and $l$ we find that the propagators are analytic in these parameters and therefore the model should

\footnotetext{
${ }^{1}$ The softness of the breaking refers here only to the power counting; indeed the complete classical action, including the gauge fixing, of the true topological model possesses an additional vectorial supersymmetry [6] which is definitely broken by the $k$ coupling
} 
go smoothly to the topological one which is known to be completely finite. In this case we find that the finiteness property does not hold since there are two renormalization constants, but the counterterms are still given by a B.R.S. cocycle.

The difference between these two ways of looking at the same classical problem becomes apparent at the quantum level since the quantum extensions under consideration are defined by different symmetries and therefore do not coincide off-shell and in the gauge fixing sectors. We have unified notations where possible and have chosen to present each approach separetely. Thus the Section "Quantum extension 1" contains the definitions, the strategy and the results concerning the softly broken model with $A_{\mu}^{a}$ of dimensionality

one, while the same arguments for the case with $A_{\mu}^{a}$ of dimensionality two reported in the Section "Quantum extension 2". The technical aspects of the treatment are collected in the Appendices, while comments on both extensions are contained in the conclusive Section.

\section{Quantum extension 1}

We start with the classical action

$$
I_{0}=-\frac{1}{2} \int d^{4} x\left(\varepsilon^{\mu \nu \rho \sigma} F_{\mu \nu}^{a} B_{\rho \sigma}^{a}+k A_{\mu}^{a} A^{a \mu}+\frac{l}{2} F^{a \mu \nu} F_{\mu \nu}^{a}\right)
$$

which is invariant (at $k=0$ ) under (3) and the gauge transformations

$$
\begin{aligned}
\delta^{(1)} A_{\mu}^{a} & =\left(D_{\mu} \theta\right)^{a} \\
\delta^{(1)} B_{\mu \nu}^{a} & =f^{a b c} B_{\mu \nu}^{b} \theta^{c}
\end{aligned}
$$

The $k$ coupling breaks softly the $\delta^{(1)}$ symmetry since

$$
\delta^{(1)} I_{0}=k \int d^{4} x \theta^{a} \partial_{\mu} A^{a \mu}
$$

This signals the fact that if we gauge fix the $\delta^{(1)}$ transformation in the Landau gauge, 
we could be able to reabsorb the breaking (9) by means of the corresponding Lagrange multiplier field, which, being free, allows a complete control of the quantum corrections.

The gauge fixing of the invariances (33;8) is by now well known [3, 8]; the corresponding non-vanishing BRS transformations are

$$
\begin{aligned}
s A_{\mu}^{a} & =\left(D_{\mu} \theta\right)^{a} \\
s \theta^{a} & =\frac{1}{2} f^{a b c} \theta^{b} \theta^{c} \\
s c_{\mu}^{a} & =\left(D_{\mu} d\right)^{a}+f^{a b c} \theta^{c} c_{\mu}^{b} \\
s d^{a} & =f^{a b c} \theta^{b} d^{c} \\
s B_{\mu \nu}^{a} & =\left(D_{\mu} c_{\nu}-D_{\nu} c_{\mu}\right)^{a}+f^{a b c} B_{\mu \nu}^{b} \theta^{c}+f^{a b c} \varepsilon_{\mu \nu \rho \sigma}\left(\partial^{\rho} \bar{c}^{b \sigma}\right) d^{c} \\
s \bar{c}_{\mu}^{a} & =b_{\mu}^{a} \\
s \bar{\theta}^{a} & =b^{a} \\
s \overline{\bar{d}}^{a} & =\bar{b}^{a} \\
s e^{a} & =\lambda^{a}
\end{aligned}
$$

The gauge fixing action is given by

$$
\begin{aligned}
I_{g f} & =\int d^{4} x\left(b^{a} \partial_{\mu} A^{a \mu}-\bar{\theta}^{a} \partial^{\mu}\left(D_{\mu} \theta\right)^{a}+b^{a \mu} \partial^{\nu} B_{\mu \nu}^{a}\right. \\
& -\frac{l}{8} b^{a \mu}\left(\left(\partial_{\rho} \partial^{\rho}-\frac{k}{l}\right) \delta_{\mu}^{\nu}-\partial_{\mu} \partial^{\nu}\right) b_{\nu}^{a}+b_{\mu}^{a}\left(\partial^{\mu} e^{a}\right) \\
& +\left(\partial_{\mu} \bar{c}^{a \mu}\right) \lambda^{a}-\left(\partial^{\mu} \overline{\bar{d}}^{a}\right)\left(\left(D_{\mu} d\right)^{a}+f^{a b c} \theta^{c} c_{\mu}^{b}\right) \\
& +\left(\partial^{\nu} \bar{c}^{a \mu}\right)\left(\left(D_{\mu} c_{\nu}\right)^{a}-\left(D_{\nu} c_{\mu}\right)^{a}+f^{a b c} B_{\mu \nu}^{b} \theta^{c}\right) \\
& \left.+\frac{1}{2} f^{a b c} \varepsilon^{\mu \nu \rho \sigma}\left(\partial_{\mu} \bar{c}_{\nu}^{a}\right)\left(\partial_{\rho} \bar{c}_{\sigma}^{b}\right) d^{c}+\bar{b}^{a} \partial^{\mu} c_{\mu}^{a}\right)
\end{aligned}
$$

and the external field contribution is 


$$
\begin{aligned}
I_{e f} & =\int d^{4} x\left(\gamma^{a \mu \nu}\left(s B_{\mu \nu}^{a}\right)+\gamma^{a \mu}\left(s A_{\mu}^{a}\right)+\xi^{a}\left(s \theta^{a}\right)\right. \\
& \left.+\eta^{a}\left(s d^{a}\right)+\Omega^{a \mu}\left(s c_{\mu}^{a}\right)+\frac{1}{2} f^{a b c} \varepsilon_{\mu \nu \rho \sigma} \gamma^{a \mu \nu} \gamma^{b \rho \sigma} d^{c}\right)
\end{aligned}
$$

The fact that the $s$ transformation is nilpotent only on-shell, since

$$
s^{2} B_{\mu \nu}^{a}=-f^{a b c} F_{\mu \nu}^{b} d^{c}
$$

is accounted for, in (12), by the bilinear $\gamma_{\mu \nu}^{a}$ coupling.

The quantum number assignement for the quantized fields is

\begin{tabular}{|l|r|r|r|r|r|r|r|r|r|r|r|r|r|}
\hline & $A_{\mu}$ & $B_{\mu \nu}$ & $\theta$ & $\bar{\theta}$ & $b$ & $c_{\mu}$ & $\bar{c}_{\mu}$ & $b_{\mu}$ & $d$ & $\overline{\bar{d}}$ & $\bar{b}$ & $e$ & $\lambda$ \\
\hline $\operatorname{dim}$ & 1 & 2 & 0 & 2 & 2 & 1 & 1 & 1 & 0 & 2 & 2 & 2 & 2 \\
\hline$\Phi \Pi$ & 0 & 0 & 1 & -1 & 0 & 1 & -1 & 0 & 2 & -2 & -1 & 0 & 1 \\
\hline G-parity & + & - & + & + & + & - & - & - & - & - & - & - & - \\
\hline
\end{tabular}

and for the external ones is

\begin{tabular}{|l|r|r|r|r|r|}
\hline & $\gamma_{\mu}$ & $\xi$ & $\eta$ & $\gamma_{\mu \nu}$ & $\Omega_{\mu}$ \\
\hline $\operatorname{dim}$ & 3 & 4 & 4 & 2 & 3 \\
\hline$\Phi \Pi$ & -1 & -2 & -3 & -1 & -2 \\
\hline G-parity & + & + & - & - & - \\
\hline
\end{tabular}

The classical action

$$
I^{c l}=I_{0}+I_{g f}+I_{e f}
$$

is G-parity even, $\Phi \Pi$ neutral and satisfies the softly broken BRS identity

$$
\begin{aligned}
\int d^{4} x\left(\frac{\delta I^{c l}}{\delta \gamma_{\mu}^{a}} \frac{\delta I^{c l}}{\delta A^{a \mu}}\right. & +\frac{\delta I^{c l}}{\delta \gamma_{\mu \nu}} \frac{\delta I^{c l}}{\delta B^{a \mu \nu}}+\frac{\delta I^{c l}}{\delta \xi^{a}} \frac{\delta I^{c l}}{\delta \theta^{a}}+\frac{\delta I^{c l}}{\delta d^{a}} \frac{\delta I^{c l}}{\delta \eta^{a}} \\
+\frac{\delta I^{c l}}{\delta c_{\mu}^{a}} \frac{\delta I^{c l}}{\delta \Omega^{a \mu}} & \left.+b^{a} \frac{\delta I^{c l}}{\delta \bar{\theta}^{a}}+b_{\mu}^{a} \frac{\delta I^{c l}}{\delta \bar{c}_{\mu}^{a}}+\bar{b}^{a} \frac{\delta I^{c l}}{\delta \overline{\overline{\bar{d}^{a}}}}+\lambda^{a} \frac{\delta I^{c l}}{\delta e^{a}}\right) \\
& =k \int d^{4} x \theta^{a} \partial_{\mu} A^{a \mu}
\end{aligned}
$$


the local equation of motion symmetries

$$
\begin{aligned}
T^{a}(x) I^{c l} & \equiv \frac{\delta I^{c l}}{\delta b^{a}(x)}=\partial_{\mu} A^{a \mu}(x) \\
\bar{T}^{a}(x) I^{c l} & \equiv \frac{\delta I^{c l}}{\delta \bar{\theta}^{a}(x)}+\partial_{\mu} \frac{\delta I^{c l}}{\delta \gamma_{\mu}^{a}(x)}=0 \\
R_{\mu}^{a}(x) I^{c l} & \equiv \frac{\delta I^{c l}}{\delta b_{\mu}^{a}(x)}=\partial^{\nu} B_{\mu \nu}^{a}(x)-\frac{l}{4}\left(\left(\partial_{\rho} \partial^{\rho}-\frac{k}{l}\right) \delta_{\mu \nu}-\partial_{\mu} \partial^{\nu}\right) b_{\nu}^{a}(x)+\partial_{\mu} e^{a}(x) \\
\bar{R}_{\mu}^{a}(x) I^{c l} & \equiv \frac{\delta I^{c l}}{\delta \bar{c}_{\mu}^{a}(x)}+\partial_{\nu} \frac{\delta I^{c l}}{\delta \gamma_{\mu \nu}^{a}(x)}=-\partial_{\mu} \lambda^{a}(x) \\
U^{a}(x) I^{c l} & \equiv \frac{\delta I^{c l}}{\delta \bar{b}^{a}(x)}=\partial_{\mu} c^{a \mu} \\
\bar{U}^{a}(x) I^{c l} & \equiv \frac{\delta I^{c l}}{\delta \overline{\bar{d}}^{a}(x)}-\partial_{\mu} \frac{\delta I^{c l}}{\delta \Omega^{a \mu}(x)}=0 \\
\bar{V}^{a}(x) I^{c l} & \equiv \frac{\delta I^{c l}}{\delta e^{a}(x)}=\partial_{\mu} b^{a \mu}(x) \\
V^{a}(x) I^{c l} & \equiv \frac{\delta I^{c l}}{\delta \lambda^{a}(x)}=\partial_{\mu} \bar{c}^{a \mu}(x)
\end{aligned}
$$

and the integrated $d^{a}$ ghost equation of motion [9]

$$
\begin{aligned}
N^{a} I^{c l} & \equiv \int d^{4} x\left(\frac{\delta I^{c l}}{\delta d^{a}}=-\int d^{4} x f^{a b c} \overline{\bar{d}} \frac{\delta I^{c l}}{\delta b^{c}}\right) \\
& +f^{a b c} \int d^{4} x\left(\varepsilon^{\mu \nu \rho \sigma} \gamma_{\mu \nu}^{b}\left(\partial_{\rho} \bar{c}_{\sigma}^{c}+\frac{1}{2} \gamma_{\rho \sigma}^{c}\right)+\eta^{b} \theta^{c}+\Omega^{c \mu} A_{\mu}^{b}\right)
\end{aligned}
$$

The defining symmetries $(15 ; 16 ;$ :17) are the starting point to analyze the perturbative renormalizability of the model; setting

$$
\Gamma=I^{c l}+\sum_{n=1} \Gamma^{(n)} \hbar^{n}
$$

we have that $\Gamma^{(n)}$ obeys

$$
\begin{aligned}
S_{L} \Gamma^{(n)} \equiv \int d^{4} x & \left(\frac{\delta I^{c l}}{\delta \gamma_{\mu}^{a}} \frac{\delta \Gamma^{(n)}}{\delta A^{a \mu}}+\frac{\delta \Gamma^{(n)}}{\delta \gamma_{\mu}^{a}} \frac{\delta I^{c l}}{\delta A^{a \mu}}+\frac{\delta I^{c l}}{\delta \gamma_{\mu \nu}} \frac{\delta \Gamma^{(n)}}{\delta B^{a \mu \nu}}+\right. \\
\frac{\delta \Gamma^{(n)}}{\delta \gamma_{\mu \nu}} \frac{\delta I^{c l}}{\delta B^{a \mu \nu}} & +\frac{\delta I^{c l}}{\delta \xi^{a}} \frac{\delta \Gamma^{(n)}}{\delta \theta^{a}}+\frac{\delta \Gamma^{(n)}}{\delta \xi^{a}} \frac{\delta I^{c l}}{\delta \theta^{a}}+\frac{\delta I^{c l}}{\delta d^{a}} \frac{\delta \Gamma^{(n)}}{\delta \eta^{a}} \\
+\frac{\delta \Gamma^{(n)}}{\delta d^{a}} \frac{\delta I^{c l}}{\delta \eta^{a}} & +\frac{\delta I^{c l}}{\delta c_{\mu}^{a}} \frac{\delta \Gamma^{(n)}}{\delta \Omega^{a \mu}}+\frac{\delta \Gamma^{(n)}}{\delta c_{\mu}^{a}} \frac{\delta I^{c l}}{\delta \Omega^{a \mu}} b^{a} \frac{\delta \Gamma^{(n)}}{\delta \bar{\theta}^{a}}+b_{\mu}^{a} \frac{\delta \Gamma^{(n)}}{\delta \bar{c}_{\mu}^{a}}+ \\
\bar{b}^{a} \frac{\delta \Gamma^{(n)}}{\delta \overline{\overline{d^{a}}}} & \left.+\lambda^{a} \frac{\delta \Gamma^{(n)}}{\delta e^{a}}-k \theta^{a} \frac{\delta \Gamma^{(n)}}{b^{a}}\right)
\end{aligned}
$$




$$
\begin{aligned}
T^{a}(x) \Gamma^{(n)} & =0 \\
\bar{T}^{a}(x) \Gamma^{(n)} & =0 \\
R_{\mu}^{a}(x) \Gamma^{(n)} & =0 \\
\bar{R}_{\mu}^{a}(x) \Gamma^{(n)} & =0 \\
U^{a}(x) \Gamma^{(n)} & =0 \\
\bar{U}^{a}(x) \Gamma^{(n)} & =0 \\
\bar{V}^{a}(x) \Gamma^{(n)} & =0 \\
V^{a}(x) \Gamma^{(n)} & =0 \\
N^{a} \Gamma^{(n)} & =0
\end{aligned}
$$

The linearized operator $S_{L}$ in (19) is not nilpotent due to the $k$ coupling; by direct computation we find:

$$
\begin{aligned}
S_{L}^{2} \equiv- & k \int d^{4} x\left(\partial_{\mu} \theta^{a} \frac{\delta}{\delta \gamma_{\mu}^{a}}+\partial_{\mu} A^{a \mu} \frac{\delta}{\delta \xi^{a}}\right)+ \\
& -k \int d^{4} x\left(\theta^{a}\left(\frac{\delta}{\delta \bar{\theta}^{a}}+\partial_{\mu} \frac{\delta}{\delta \gamma_{\mu}^{a}}\right)\right)-\frac{1}{2} k f^{a b c} \int d^{4} x \theta^{b} \theta^{c} \frac{\delta}{\delta b^{a}}
\end{aligned}
$$

and therefore on $\Gamma^{(n)}$ the nilpotency condition reduces to

$$
D \Gamma^{(n)} \equiv \int d^{4} x\left(\partial_{\mu} \theta^{a} \frac{\delta}{\delta \gamma_{\mu}^{a}}+\partial_{\mu} A^{a \mu} \frac{\delta}{\delta \xi^{a}}\right) \Gamma^{(n)}=0
$$

The other algebraic relations are

$$
\begin{aligned}
{\left[S_{L}, T^{a}(x)\right] } & =\bar{T}^{a}(x) \\
{\left[S_{L}, R_{\mu}^{a}(x)\right] } & =\bar{R}_{\mu}^{a}(x) \\
{\left[S_{L}, U^{a}(x)\right] } & =\bar{U}^{a}(x) \\
{\left[S_{L}, V^{a}(x)\right] } & =\bar{V}^{a}(x) \\
{\left[S_{L}, D^{a}\right] \equiv G^{a} } & =-f^{a b c} \int d^{4} x\left(\eta^{b} \frac{\delta}{\delta \xi^{c}}+\Omega_{\mu}^{b} \frac{\delta}{\delta \gamma_{\mu}^{c}}\right) \\
& -f^{a b c} \int d^{4} x\left(\frac{1}{4} \varepsilon^{\mu \nu \rho \sigma} \gamma_{\mu \nu}^{c} \frac{\delta}{\delta B^{b \rho \sigma}}+\theta^{b} \frac{\delta}{\delta d^{c}}+A_{\mu}^{b} \frac{\delta}{\delta c_{\mu}^{c}}\right)
\end{aligned}
$$


The analysis of the quantum extension of the symmetries identifing the model begins with the functional relations $(20)$ which imply that $\Gamma^{(n)}$ is independent of the fields $b^{a}(x), b_{\mu}^{a}(x), e^{a}(x), \lambda^{a}(x), \bar{b}^{a}(x)$ and it depends on the external fields $\gamma_{\mu}^{a}, \gamma_{\mu \nu}^{a}, \Omega_{\mu}^{a}$ and the antighosts $\bar{\theta}^{a}(x), \bar{c}_{\mu}^{a}(x), \overline{\bar{d}}^{a}(x)$ through the linear combinations

$$
\begin{aligned}
\widehat{\gamma}_{\mu}^{a}(x) & =\gamma_{\mu}^{a}(x)+\partial_{\mu} \bar{\theta}^{a}(x) \\
\widehat{\gamma}_{\mu \nu}^{a}(x) & =\gamma_{\mu \nu}^{a}(x)+\frac{1}{2}\left(\partial_{\mu} \bar{c}_{\nu}^{a}(x)-\partial_{\nu} \bar{c}_{\mu}^{a}(x)\right) \\
\widehat{\Omega}_{\mu}^{a}(x) & =\Omega_{\mu}^{a}(x)-\partial_{\mu} \overline{\bar{d}}^{a}(x)
\end{aligned}
$$

while $d^{a}(x)$ appears only as $\partial_{\mu} d^{a}(x)$. Substituting the relations (20) into (19) we obtain the identity

$$
\begin{aligned}
\widehat{S}_{L} \Gamma^{(n)} \equiv \int d^{4} x & \left(\frac{\delta \widehat{I}}{\delta \widehat{\gamma}_{\mu}^{a}} \frac{\delta \Gamma^{(n)}}{\delta A^{a \mu}}+\frac{\delta \Gamma^{(n)}}{\delta \widehat{\gamma}_{\mu}^{a}} \frac{\delta \widehat{I}}{\delta A^{a \mu}}+\frac{\delta \widehat{I}}{\delta \widehat{\gamma}_{\mu \nu}} \frac{\delta \Gamma^{(n)}}{\delta B^{a \mu \nu}}\right. \\
+ & \frac{\delta \Gamma^{(n)}}{\delta \widehat{\gamma}_{\mu \nu}} \frac{\delta \widehat{I}}{\delta B^{a \mu \nu}}+\frac{\delta \widehat{I}}{\delta \xi^{a}} \frac{\delta \Gamma^{(n)}}{\delta \theta^{a}}+\frac{\delta \Gamma^{(n)}}{\delta \xi^{a}} \frac{\delta \widehat{I}}{\delta \theta^{a}}+\frac{\delta \widehat{I}}{\delta d^{a}} \frac{\delta \Gamma^{(n)}}{\delta \eta^{a}} \\
& \left.+\frac{\delta \Gamma^{(n)}}{\delta d^{a}} \frac{\delta \widehat{I}}{\delta \eta^{a}}+\frac{\delta \widehat{I}}{\delta c_{\mu}^{a}} \frac{\delta \Gamma^{(n)}}{\delta \widehat{\Omega}^{a \mu}}+\frac{\delta \Gamma^{(n)}}{\delta c_{\mu}^{a}} \frac{\delta \widehat{I}}{\delta \widehat{\Omega}^{a \mu}}\right)
\end{aligned}
$$

where

$$
\begin{aligned}
\widehat{I} & =-\frac{1}{2} \int d^{4} x\left(\varepsilon^{\mu \nu \rho \sigma} F_{\mu \nu}^{a} B_{\rho \sigma}^{a}+k A_{\mu}^{a} A^{a \mu}+\frac{1}{2} F^{a \mu \nu} F_{\mu \nu}^{a}\right) \\
& +\int d^{4} x\left(\widehat{\gamma}^{a \mu \nu}\left(\left(D_{\mu} c_{\nu}-D_{\nu} c_{\mu}\right)^{a}-f^{a b c} B_{\mu \nu}^{b} \theta^{c}\right)+\widehat{\gamma}^{a \mu}\left(D_{\mu} \theta\right)^{a}+\xi^{a} \frac{1}{2} f^{a b c} \theta^{b} \theta^{c}\right. \\
& \left.+\eta^{a} f^{a b c} \theta^{b} d^{c}+\widehat{\Omega}^{a \mu}\left(\left(D_{\mu} d\right)^{a}+f^{a b c} \theta^{b} c_{\mu}^{c}\right)+\frac{1}{2} f^{a b c} \varepsilon_{\mu \nu \rho \sigma} \widehat{\gamma}^{a \mu \nu} \widehat{\gamma}^{b \rho \sigma} d^{c}\right)
\end{aligned}
$$

In Appendix B we discuss the renormalization of the nilpotency condition

$$
\widehat{D} \Gamma^{(n)} \equiv \int d^{4} x\left(\partial_{\mu} \theta^{a} \frac{\delta}{\delta \widehat{\gamma}_{\mu}^{a}}+\partial_{\mu} A^{a \mu} \frac{\delta}{\delta \xi^{a}}\right) \Gamma^{(n)}=0
$$

By restricting the space to functionals obeying (27) we have a nilpotent $\widehat{S}_{L}$ operator and in order to analyze the stability and the absence of anomalies of the model we have 
to identify the integrated cohomology spaces of $\widehat{S}_{L}$ in the sectors with $\Phi \Pi$ charge zero and one.

The integrated cohomology is identified through the descent system [10]

$$
\begin{aligned}
\widehat{S}_{L} X_{p}^{4}(x) & =\partial^{\mu}\left(X_{\mu}\right)_{p+1}^{3}(x) \\
\widehat{S}_{L}\left(X_{\mu}\right)_{p+1}^{3}(x) & =\partial^{\nu}\left(X_{[\mu \nu]}\right)_{p+2}^{2}(x) \\
\widehat{S}_{L}\left(X_{[\mu \nu]}\right)_{p+2}^{2}(x) & =\partial^{\rho}\left(X_{[\mu \nu \rho]}\right)_{p+3}^{1}(x) \\
\widehat{S}_{L}\left(X_{[\mu \nu \rho]}\right)_{p+3}^{1}(x) & =\partial^{\sigma}\left(X_{[\mu \nu \rho \sigma]}\right)_{p+4}^{0}(x) \\
\widehat{S}_{L}\left(X_{[\mu \nu \rho \sigma]}\right)_{p+4}^{0}(x) & =0
\end{aligned}
$$

where the upper index denotes the dimensionality and the lower one the $\Phi \Pi$ charge, hence we will be interested to the cases $p=0,1$. We emphasize that the analysis is not to be performed in the space of forms since the classical action does not belong to this space due to the presence of the terms $\int d^{4} x k A_{\mu}^{a} A^{a \mu}$ and $\int d^{4} x F^{a \mu \nu} F_{\mu \nu}^{a}$.

The detailed discussion of the local cohomology spaces is performed in Appendix $\mathrm{C}$, the results can be summarized by saying that the local functions $X^{c h}(x)$ of canonical dimension $\leq 4$, even G-parity, satisfying the constraint (22) and which are cocycles modulo coboundaries of $\widehat{S}_{L}$ are the gauge invariant functions of the sole field $\theta^{a}$ without space-time derivatives.

It is now easy to reconstruct the solution of the descent equations (28) since in the case $p=0,1$ the $X^{c h}(x)$ terms are absent due to power counting and Lorentz indices content; thus we conclude that

$$
X_{0,1}^{4}(x)=\widehat{S}_{L} X_{-1,0}^{4}(x)+\partial^{\mu} X_{\mu 0,1}^{3}(x)
$$

and therefore

$$
X_{0,1}^{4}=\int d^{4} x X_{-1,0}^{4}(x)=\widehat{S}_{L} \int d^{4} x X_{-1,0}^{4}(x)
$$

This result, which implies the absence of anomalies, also tells us that all counterterms of the model are BRS variations of local terms. Their number can be computed by 
parametrizing the general local functional $\widehat{\Gamma}^{(0)}$ of dimensions $\leq 4$, even G-parity, $\Phi \Pi$ charge -1 and obeying

$$
\begin{aligned}
\widehat{D} \widehat{\Gamma}^{(0)} & =0 \\
G^{a} \widehat{\Gamma}^{(0)} & =0 \\
\widehat{\Gamma}^{0} & \neq \widehat{S}_{L} \widehat{\Gamma}^{0}
\end{aligned}
$$

We find that there are only two nonvanishing parameters in $\widehat{\Gamma}^{0}$; these can be identified with the multiplicative renormalization constants of the fields $A_{\mu}^{a}(x), \theta^{a}(x)$.

\section{Quantum Extension 2}

The classical action

$$
\Gamma^{c l}=-\frac{1}{2} \int d^{4} x\left[\epsilon_{\mu \nu \rho \sigma} B_{\mu \nu}^{a}(x) F_{\rho \sigma}^{a}(x)+A_{\mu}^{a}(x) A_{\mu}^{a}(x)\right]
$$

is invariant under the transformations

$$
\begin{aligned}
\delta B_{\mu \nu}^{a}(x) & =\left(\left(D_{\mu} c_{\nu}(x)-D_{\nu} c_{\mu}(x)\right)^{a}+\theta\left(g f^{a b c} F_{\mu \nu}^{b}(x) c^{c}(x)\right)\right. \\
\delta c_{\rho}^{a}(x) & =\left(D_{\rho} c(x)\right)^{a} \\
\delta \theta & =-1
\end{aligned}
$$

i.e. in terms of the operator

$$
\begin{aligned}
\delta_{B . R . S .} & =\int d^{4} x\left[\left(\left(D_{\mu} c_{\nu}(x)-D_{\nu} c_{\mu}(x)\right)^{a}+\theta g f^{a b c} F_{\mu \nu}^{b}(x) c^{c}(x)\right) \frac{\delta}{\delta B_{\mu \nu}^{a}(x)}\right. \\
& \left.+\left(D_{\rho} c(x)\right)^{a} \frac{\delta}{\delta c_{\rho}^{a}(x)}\right]-\frac{\partial}{\partial \theta}
\end{aligned}
$$

Remark that without the $\theta$ term the B.R.S. operator is nilpotent only on-shell, so we obtain an "open gauge algebra" [13] definition of the model; the introduction of this ghost 
(as a parameter carrying a negative unit of $\Phi \Pi$ charge) must be considered as a "trick" intended to insure the off-shell extension of the nilpotency condition, while it is required not to modify the dynamics described by the classical action 14; hence

$$
\begin{aligned}
\delta_{\text {B.R.S. } \Gamma^{C l}} & =0 \\
\frac{\partial \Gamma^{C l}}{\partial \theta} & =0 \\
\delta_{\text {B.R.S. }}^{2} & =0
\end{aligned}
$$

To gauge fix the invariance (33) in the presence of the $\theta$ parameter we introduce the gauge functions $G_{\mu}^{a}$, Lagrange multipliers $b_{\mu}^{a}$ and antighosts $\bar{c}_{\mu}^{a}, \bar{c}^{a}$

$$
\begin{aligned}
G_{\mu}^{a}(x) & =\left(D^{\nu} B_{\mu \nu}(x)\right)^{a}+b_{\mu}^{a}(x) \\
\delta \bar{c}_{\mu}^{a}(x) & =G_{\mu}^{a}(x) \\
\delta \bar{c}^{a}(x) & =\left(D^{\mu} \bar{c}_{\mu}(x)\right)^{a}
\end{aligned}
$$

and the gauge fixing action

$$
\Gamma^{\text {Gauge }}=\int d^{4} x\left(\frac{1}{2} G_{\mu}^{a}(x) G^{a \mu}(x)-\bar{c}_{\mu}^{a}(x)\left(D^{\mu} D^{\nu} c_{\nu}(x)\right)^{a}+\bar{c}^{a}(x)\left(D^{\nu} D_{\nu} c(x)\right)^{a}\right)
$$

The $\delta$ variation of the Lagrange multiplier is defined as

$$
\delta b_{\mu}^{a}(x)=-\left(D_{\mu} D^{\nu} c_{\nu}(x)\right)^{a}-\left(D^{\nu} D_{\mu} c_{\nu}(x)-D^{\nu} D_{\nu} c_{\mu}(x)\right)^{a}+\left(\theta D^{\nu} F_{\mu \nu}(x)\right)^{a}
$$

which yields

$$
\delta G_{\mu}^{a}(x)=-\left(D_{\mu} D^{\nu} c_{\nu}(x)\right)^{a}
$$

With this choice we have at the classical level the functional relations

$$
\frac{\delta \Gamma}{\delta \bar{c}_{\mu}^{a}(x)}=\delta G^{a \mu}(x)
$$




$$
\begin{aligned}
\frac{\delta \Gamma}{\delta b_{\mu}^{a}(x)} & =G^{a \mu}(x) \\
D_{\mu} \frac{\delta \Gamma}{\delta \bar{c}^{a}(x)} & =\delta^{2} b_{\mu}^{a}(x)=\left(D_{\mu} D^{\nu} D_{\nu} c(x)\right)^{a}
\end{aligned}
$$

which imply the nilpotency of the $\delta_{\text {B.R.S. }}$ operator. In order to transfer this framework at the quantum level we should introduce external field couplings for all the B.R.S. variations of the fields; it is well known that the procedure also implements the relations (40) and allows a complete control of the vertex functional as far as the dependence on the gauge fixing fields is concerned. Taking into account this considerable simplification we add only the sources needed to discuss the residual symmetry, hence we define

$$
\Gamma^{0}=\Gamma^{C l}+\int d^{4} x\left[\gamma^{a \mu \nu}(x) \delta B_{\mu \nu}^{a}(x)+\zeta^{a \rho}(x) \delta c_{\rho}^{a}(x)\right]
$$

and the linearized, nilpotent B.R.S. operator we consider is

$$
\begin{aligned}
\delta_{\text {B.R.S. }} & =\int d^{4} x\left[\frac{\delta \Gamma^{0}}{\delta \gamma^{a \mu \nu}(x)} \frac{\delta}{\delta B_{\mu \nu}^{a}(x)}+\frac{\delta \Gamma^{0}}{\delta B_{\mu \nu}^{a}(x)} \frac{\delta}{\delta \gamma^{a \mu \nu}(x)}\right. \\
& \left.+\frac{\delta \Gamma^{0}}{\delta \zeta^{a \nu}(x)} \frac{\delta}{\delta c_{\nu}^{a}(x)}+\frac{\delta \Gamma^{0}}{\delta c_{\nu}^{a}(x)} \frac{\delta}{\delta \zeta^{a \nu}(x)}\right]-\frac{\partial}{\partial \theta}
\end{aligned}
$$

If we factorize the $\theta$ dependent part, we can write it as:

$$
\delta_{\text {B.R.S. }} \equiv \delta_{0}+\theta \delta_{1}-\frac{\partial}{\partial \theta}
$$

and the nilpotency condition gives the algebraic rules

$$
\begin{array}{r}
\delta_{1}=\delta_{0}^{2} \\
\delta_{1} \delta_{0}=\delta_{0} \delta_{1}
\end{array}
$$

Our purpose will be to extend to the quantum level the symmetry described by (3); according to the Q.A.P. [11] for an arbitrary choice of the effective Lagrangian, the obstruction in minimal order is given by:

$$
\delta \Gamma=\Delta
$$


where $\Delta$ is a local functional decomposed into its external fields and $\theta$ dependent part as:

$$
\begin{aligned}
\Delta & \equiv \int d^{4} x\left[\Delta_{0}(x)+\theta \Delta_{1}(x)\right] \\
& +\int d^{4} x\left[\gamma^{a \mu \nu}(x)\left(\Theta_{\mu \nu 0}^{a}(x)+\theta \Theta_{\mu \nu 1}^{a}(x)\right)+\zeta^{a \rho}(x)\left(\Psi_{\rho 0}^{a}(x)\right.\right. \\
& \left.\left.+\theta \Psi_{\rho 1}^{a}(x)\right)\right]
\end{aligned}
$$

The renormalization program will be completed if the obstruction $\Delta$ in (46) can be compensated by means of local counterterms to be introduced in the effective Lagrangian. Recall that the definition of the classical model allows $\theta$ dependent countertems only in that part of the action which contains external fields, for this reason we decompose:

$$
L_{e f f}=L_{e f f}^{0}+\gamma^{a \mu \nu}(x)\left(\hat{\Theta}_{\mu \nu 0}^{a}(x)+\theta \hat{\Theta}_{\mu \nu 1}^{a}(x)\right)+\zeta^{a \rho}(x) \hat{\Psi}_{\rho 0}^{a}(x)
$$

and as subsidiary condition, we impose:

$$
\frac{\partial L_{e f f}^{0}}{\partial \theta}=0
$$

The power counting, Gparity and $\Phi \Pi$ Charge conservations control the quantum numbers of the anomaly $\Delta$ and yield contraints to the renormalization procedure; for this reason we shall summarize in the following table all the global properties of the constituent fields of the model: 


\begin{tabular}{||l|l|l|l|}
\hline \hline Fields and parameters & Dimension & $\Phi \Pi$ Charge & Gparity \\
\hline $\mathrm{g}$ & -1 & 0 & \\
\hline$B_{\mu \nu}^{i}(x)$ & 1 & 0 & - \\
\hline$A_{\mu}^{i}(x)$ & 2 & 0 & + \\
\hline$D_{\mu}^{i j}(x)$ & 1 & 0 & + \\
\hline$F_{\mu \nu}^{i}(x)$ & 3 & 0 & + \\
\hline$C_{\sigma}^{i}(x)$ & 1 & 1 & - \\
\hline$C^{i}(x)$ & 1 & 2 & - \\
\hline $\bar{c}_{\sigma}^{i}(x)$ & 1 & -1 & - \\
\hline $\bar{c}^{i}(x)$ & 1 & -2 & - \\
\hline$b_{\sigma}^{i}(x)$ & 2 & 0 & - \\
\hline$\theta$ & -1 & -1 & + \\
\hline$\gamma_{i}^{\mu \nu}(x)$ & 2 & -1 & - \\
\hline$\zeta_{i}^{\rho}(x)$ & 2 & -2 & - \\
\hline$\hat{\Theta}_{\mu \nu 0}^{i}(x)$ & 2 & 1 & - \\
\hline$\Theta_{\mu \nu 1}^{i}(x)$ & 3 & 2 & - \\
\hline$\hat{\Psi}_{\rho 0}^{i}$ & 2 & 2 & - \\
\hline$\Delta_{0}(x)$ & 5 & 1 & + \\
\hline$\Delta_{1}(x)$ & 6 & 2 & + \\
\hline$\Theta_{\mu \nu 0}^{i}(x)$ & 3 & 2 & - \\
\hline$\Theta_{\mu \nu 1}^{i}(x)$ & 4 & 3 & - \\
\hline$\Psi_{\rho 0}^{i}(x)$ & 3 & 3 & - \\
\hline$\Psi_{\rho 1}^{i}(x)$ & 4 & 4 & - \\
\hline
\end{tabular}

Now the external fields anomalies are absent since we shall see that the consistency (cocycle) condition indeed coincides with compensability (cobordism) so this part of the cohomology will be empty from the very definition.

In fact the cocycle condition:

$$
\delta_{\text {B.R.S. }} \Delta=0
$$

requires:

$$
\begin{aligned}
\Psi_{\rho 1}^{i}(x) & =\delta_{0} \Psi_{\rho 0}^{i}(x) \\
\Theta_{\mu \nu 1}^{i}(x) & \left.=\delta_{0} \Theta_{\mu \nu 0}^{i}(x)\right)
\end{aligned}
$$

On the other hand the compensability condition

$$
\Delta=\delta_{\text {B.R.S. }} \hat{\Delta}
$$


written in terms of the quantities defined in (47) imposes

$$
\begin{aligned}
\Psi_{\rho 1}^{a}(x) & =\delta_{1} \hat{\Psi}_{\rho}^{a}(x) \\
\Psi_{\rho 0}^{a}(x) & =\delta_{0} \hat{\Psi}_{\rho}^{a}(x) \\
\Theta_{\mu \nu 0}^{a}(x) & =\delta_{0} \hat{\Theta}_{\mu \nu 0}^{a}(x)-\hat{\Theta}_{\mu \nu 1}^{a}(x) \\
\Theta_{\mu \nu 1}^{a}(x) & =\delta_{1} \hat{\Theta}_{\mu \nu 0}^{a}(x)-\delta_{0} \hat{\Theta}_{\mu \nu 1}^{a}(x)
\end{aligned}
$$

It is straightforward to check, eliminating the hatted fields in (52) by means of the relations (44), that we reconstruct the cocycle conditions: therefore this cohomology subspace is empty.

We consider now the external field independent part of the anomaly which is analyzed according to the $\theta$ dependence as

$$
\begin{aligned}
& \int d^{4} x \Delta_{1}(x)=\delta_{1} \Gamma \\
& \int d^{4} x \Delta_{0}(x)=\delta_{0} \Gamma
\end{aligned}
$$

The cocycle (consistency) condition (48) implies:

$$
\begin{aligned}
\int d^{4} x \Delta_{1}(x) & =\delta_{0} \int d^{4} x \Delta_{0}(x) \\
\delta_{0} \int d^{4} x \Delta_{1}(x) & =\delta_{1} \int d^{4} x \Delta_{0}(x)
\end{aligned}
$$

Decomposing the anomalies according to their $\Phi$.П. charge content we obtain:

$$
\begin{aligned}
\int d^{4} x \Delta_{1}(x) & =\int d^{4} x\left[c^{a}(x) \Delta_{1, a}(x)+\sum_{m} c_{\mu}^{a}(x) \partial_{\lambda_{m}}{ }^{m} c_{\nu}^{b}(x) \Delta_{1, a, b, \lambda_{m}}^{\mu \nu}(x)\right] \\
\int d^{4} x \Delta_{0}(x) & =\int d^{4} x\left[c_{\mu}^{a}(x) \Delta_{0, a}^{\mu}(x)\right]
\end{aligned}
$$

where $\Delta_{0, a}^{\mu}(x), \Delta_{1, a}(x)$ and $\Delta_{1, a, b, \lambda_{m}}^{\mu \nu}(x)$ are $\Phi . \Pi$. neutral.

The first cocycle condition(54) furthermore gives:

$$
\begin{aligned}
& \int d^{4} x\left[c^{a}(x) \Delta_{1, a}(x)+\sum_{m} c_{\mu}^{a}(x) \partial_{\lambda_{m}}{ }^{m} c_{\nu}^{b}(x) \Delta_{1, a, b, \lambda_{m}}^{\mu \nu}(x)\right] \\
& =-\int d^{4} x\left[c^{a}(x)\left(D_{\mu a}^{b} \Delta_{0 b}^{\mu}(x)\right)+c_{\mu}^{a}(x) \delta_{0} \Delta_{0, a}^{\mu}(x)\right]
\end{aligned}
$$


and consequently the relations:

$$
\begin{aligned}
\Delta_{1, a}(x) & =-\left(D_{\mu a}^{b} \Delta_{0 b}^{\mu}(x)\right) \sum_{m}-1^{m}\left(\partial_{\lambda_{m}}{ }^{m} \Delta_{1, a, b, \lambda_{m}}^{\mu \nu}(x)-(\mu \rightarrow \nu)(i \rightarrow j)\right) \\
& =D_{\rho} \frac{\delta}{\delta B_{\rho \nu}^{b}(x)} \int d^{4} y \Delta_{0, a}^{\mu}(y)-\int d^{4} y D_{\rho}^{y} \frac{\delta}{\delta B_{\rho \nu}^{b}(y)} \Delta_{0, a}^{\nu}(x)
\end{aligned}
$$

and the $c^{a}(x)$ content of $\Delta_{1, a}(x)$ disappears if and only if $\left(D_{\mu a}^{b} \Delta_{0 b}^{\mu}(x)\right)=0$.

This step allows us to considerably simplify our treatment in terms of local quantities; indeed the model is defined in a vector space whose entities are the monomials of local constituent fields and their space-time derivatives considered as independent.

It is useful to introduce in this space a new coordinate system, for the uncharged $\Phi . \Pi$. sector, such that each function of the fields and their space-time derivatives is reparametrized in a different way. If we grade this space in terms of ordinary space-time derivatives, it is a trivial observation that a monomial containing a space-time derivative of order $\mathrm{n}$ can be written in terms of one containing covariant derivatives of the same order plus terms of lower order in the covariant derivatives .

In this naive decomposition we have to take into account that while in each monomial written in terms of the ordinary space-time derivatives, the derivatives tensorial content is by construction completely symmetrized, this is not so for the same quantities written in terms of covariant derivatives. Thus, to implement this symmetrization, we are compelled to introduce the curvature tensor $g F_{\mu \nu}^{j}(x)$ and in general the symmetrized covariant derivatives of $\left.g F_{\mu \nu}^{j}(x)\right)$ as independent fields .

This implies a one-to-one map between the space of monomials of ordinary space-time derivatives of fields and that given by symmetrized covariant derivatives (which we shall denote by $\left.\left\{D_{\alpha}\right\}_{n}\right)$ of the same fields plus symmetrized covariant derivatives of $g F_{\mu \nu}^{j}(x)$, considered as independent fields.

This notation has the further advantage : the coupling costant $\mathrm{g}$ (which has a negative dimension and so its presence may cause problems with a lower limit in the power counting analysis) is contained explicitely at the classical level in the covariant derivatives and in 
the curvature tensor $g F_{\mu \nu}^{j}(x)$; in the new basis the coupling constant will disappear from the B.R.S. operator, while in the action it is present at the tree level as an inverse power $\frac{1}{g}$ (which has positive power counting dimension).

In this framework, defining

$$
L^{a}(x)=f^{a b c} \sum_{n}\left\{D_{\alpha}\right\}_{n} g F_{\mu \nu}^{b}(x) \frac{\delta}{\delta\left\{D_{\alpha}\right\}_{n} B_{\mu \nu}^{c}}
$$

the Q.A.P. gives for $\Delta_{1, a}(x)$

$$
L^{a}(x) \Gamma=\Delta_{1, a}(x)
$$

and $\Delta_{1, a}(x)$ has dimension 5 and odd Gparity .

Defining:

$$
L^{a \dagger}(x)=f^{a b c} \sum_{n}\left\{D_{\alpha}\right\}_{n} B_{\mu \nu}^{c} \frac{\delta}{\delta\left\{D_{\alpha}\right\}_{n} g F_{\mu \nu}^{b}(x)}
$$

we compute the commutators:

$$
\begin{aligned}
{\left[L^{a}(x), L^{a \dagger}(x)\right]_{-} } & =\sum_{n}\left\{D_{\alpha}\right\}_{n} g F_{\rho \sigma}^{b}(x) \frac{\delta}{\left\{D_{\alpha}\right\}_{n} g F_{\rho \sigma}^{b}(x)} \\
& -\sum_{n}\left\{D_{\alpha}\right\}_{n} B_{\rho \sigma}^{b}(x) \frac{\delta}{\left\{D_{\alpha}\right\}_{n} B_{\rho \sigma}^{b}(x)}=\left(N_{g F}-N_{B}\right) \equiv S(x) \\
{\left[L^{a}(x), S(x)\right]_{-} } & =-2 L^{a}(x)
\end{aligned}
$$

The above relations yield the identitis:

$$
\begin{aligned}
{\left[L^{a}(x), S(x)\right]_{-} \Gamma } & =-2 L^{a}(x) \Gamma \\
\left(N_{g F}-N_{B}-2\right) \Delta_{1, a}(x) & =L^{a}(x) S(x) \Gamma
\end{aligned}
$$

and each monomial $\Delta_{1, a}(x)$ such that

$$
\left(N_{g F}-N_{B}-2\right) \Delta_{1, a}(x) \neq 0
$$

clearly admits a counterterm compensation. 
After this step we are left with the possible breakings satisfying

$$
\left(N_{g F}-N_{B}-2\right) \Delta_{1, a}(x)=0
$$

which can be parametrized as

$$
\Delta_{1, a}(x)=\Lambda_{a b c 0} S^{\mu \nu \rho \sigma \lambda} D_{\lambda} F_{\mu \nu}^{b}(x) F_{\rho \sigma}^{c}(x)+\frac{1}{g} \Lambda_{a b c 1} S_{\mu \nu \rho \sigma} g F_{\mu \nu}^{b}(x) g F_{\rho \sigma}^{c}(x)
$$

where $\Lambda_{a b c 0}, \Lambda_{a b c 1}, S_{\mu \nu \rho \sigma \lambda}$ and $S_{\mu \nu \rho \sigma}$ are dimensionless group and Lorentz invariant tensors respectively.

Now $S_{\mu \nu \rho \sigma \lambda}$ vanishes since no dimensionless Lorentz invariant tensor with odd indices exists in four dimensions, and the odd Gparity requirement forces $S_{\mu \nu \rho \sigma}=\epsilon_{\mu \nu \rho \sigma} \Lambda_{a b c}=d_{a b c}$ where $d_{a b c}$ is the symmetric group invariant tensor, so we are left with:

$$
\Delta_{1, a}(x)=a_{1} \frac{1}{g} d_{a b c} \epsilon_{\mu \nu \rho \sigma} g F_{\mu \nu}^{b}(x) g F_{\rho \sigma}^{c}(x)
$$

Finally the expression in (66) does not satisfy the consistency condition (57) since:

$$
\begin{aligned}
\Delta_{1, a}(x) & =a_{1} D_{\mu d}^{f}\left[\frac{1}{g} d_{a b c} f_{b f e} \epsilon_{\mu \nu \rho \sigma} D_{\nu e}^{d} g F_{\rho \sigma}^{c}(x)\right] \\
& \neq-\left(D_{\mu a}^{b} \Delta_{0 b}^{\mu}(x)\right)
\end{aligned}
$$

and therefore the coefficient $a_{1}$ must vanish.

By (57), the vanishing of $\Delta_{1, a}$ implies for $\Delta_{0, a}^{\mu}$ the constraints

$$
\left(D_{\mu a}^{b} \Delta_{0 b}^{\mu}(x)\right) \equiv \partial_{\mu} \Delta_{0 a}^{\mu}(x)-g f^{a b c} A_{\mu}^{c} \Delta_{0 b}^{\mu}(x)=0
$$

and:

$$
L^{a}(x) \Delta_{0 b}^{\mu}(x)=0
$$

which have to be verified for $\Delta_{0 b}^{\mu}(x)$ of dimension 4 and even Gparity.

This last equation admits the solution:

$$
\frac{\delta}{\delta\left\{D_{\alpha}\right\}_{n} B_{\mu \nu}^{c}} \Delta_{0 b}^{\rho}(x)=\left\{D_{\alpha}\right\}_{n} g F_{\mu \nu}^{c}(x) X_{\rho}(A, g F)^{-}+\left\{D_{\alpha}\right\}_{n} \epsilon^{\mu \nu \lambda \eta} g F_{\lambda \eta}^{c}(x) X_{\rho}(A, g F)^{+}
$$


where $X_{\rho}(A, g F)^{+} X_{\rho}(A, g F)^{-}$are arbitrary analytic functions of $(A, g F)$ of even $(+)$ or odd(-) G-parity. Dimensional analysis points to $n=0$ as the only possibility, but in this case the $X_{\rho}$ functions have dimension one, which is not satisfiable with their field content.

This argument excludes the anomalies:

$$
\Delta_{1}=\int d^{4} x\left[\sum_{m} c_{\mu}^{a}(x) \partial_{\lambda_{m}} c_{\nu}^{b}(x) \Delta_{1, a, b, \lambda_{m}}^{\mu \nu}(x)\right]
$$

and we remain with the case when $\Delta_{0 b}^{\mu}(x)$, considered as formal power series in $1 / \mathrm{g}$, is $B_{\mu \nu}^{a}$ independent and depends only on the $A_{\mu}^{a}, g F_{\mu \nu}^{c}(x)$ fields and their covariant derivatives. Dimensional analysis, G-parity and Lorentz index content easily imply that it is impossile to construct a $\Delta_{0 b}^{\mu}(x)$ which obeys (68), therefore no anomaly can occur in this model.

\section{Conclusions}

We have completely characterized the antisymmetric tensor field models in the two regimes; in particular for the "Quantum extension 1" we have proved that the integrated cohomology space of the corresponding B.R.S. operator is empty both in the neutral $\Phi \Pi$ sector and in the anomaly sector. The first property, characteristic of the topological theories, here is not sufficient to insure finiteness due to the lack of the vectorial supersymmetry and instead we find that there are two renormalization constants.

Concerning the "Quantum extension 2" where the analysis performed by means of the $\theta$ parameter offers an alternative to the better known "higher powers in the external fields' technique, we also find that there are no anomalies, thus clarifying the possibility of maintaining to all perturbative orders the $\sigma$ model interpretation . 


\section{Appendix A}

We report here the propagators of the gauge fields since they differ, due to the presence of the $\int d^{4} x F^{a \mu \nu} F_{\mu \nu}^{a}$, from the usual case: selecting the bilinear part of the classical action (14) and considering only the terms involving the fields $A_{\mu}^{a}(p), B_{\rho \sigma}^{a}(p), b^{a}(p), b_{\lambda}^{a}(p), e^{a}(p)$ we have to compute the inverse of the matrix, with rows and columns ordered accordingly,

$$
\left(\begin{array}{ccccc}
-\left(l p^{2}+k\right) \delta_{\mu \nu}+l p_{\mu} p_{\nu} & i \varepsilon_{\rho \sigma \tau \mu} p^{\tau} & -i p_{\mu} & 0 & 0 \\
-i \varepsilon_{\rho^{\prime} \sigma^{\prime} \tau^{\prime} \nu} p^{\tau} & 0 & 0 & \frac{i}{2}\left(\delta_{\rho^{\prime} \lambda^{\prime}} p_{\sigma^{\prime}}-\delta_{\sigma^{\prime} \lambda^{\prime}} p_{\rho^{\prime}}\right) & 0 \\
i p_{\nu} & 0 & 0 & 0 & 0 \\
0 & -\frac{i}{2}\left(\delta_{\rho \lambda} p_{\sigma}-\delta_{\sigma \lambda} p_{\rho}\right) & 0 & -\frac{1}{4}\left(l p^{2}+k\right) \delta_{\lambda \lambda^{\prime}}+\frac{l}{4} p_{\lambda} p_{\lambda^{\prime}} & -i p_{\lambda} \\
0 & 0 & 0 & i p_{\lambda^{\prime}} & 0
\end{array}\right)
$$

The inverse is given by

$$
\left(\begin{array}{ccccc}
0 & \frac{i}{2 p^{2}} \varepsilon_{\rho \sigma \tau \mu} p^{\tau} & -\frac{i}{p^{2}} p_{\mu} & 0 & 0 \\
-\frac{i}{2 p^{2}} \varepsilon_{\rho^{\prime} \sigma^{\prime} \tau^{\prime} \nu^{\prime}} p^{\tau^{\prime}} & \frac{\left(l p^{2}+k\right)}{4 p^{2}}\left(\delta_{\rho \rho^{\prime}} \delta_{\sigma \sigma^{\prime}}-\delta_{\rho \sigma^{\prime}} \delta_{\sigma \rho^{\prime}}\right) & 0 & \frac{i}{p^{2}}\left(\delta_{\rho^{\prime} \lambda^{\prime}} p_{\sigma^{\prime}}-\delta_{\sigma^{\prime} \lambda^{\prime}} p_{\rho^{\prime}}\right) & 0 \\
\frac{i}{p^{2}} p_{\nu^{\prime}} & 0 & \frac{k}{p^{2}} & 0 & 0 \\
0 & -\frac{i}{p^{2}}\left(\delta_{\rho \lambda} p_{\sigma}-\delta_{\sigma \lambda} p_{\rho}\right) & 0 & 0 & -\frac{i}{p^{2}} p_{\lambda} \\
0 & 0 & 0 & \frac{i}{p^{2}} p_{\lambda^{\prime}} & \frac{k}{4 p^{2}}
\end{array}\right)
$$

From the above we obtain the U.V. dimensions of the gauge fields reported in text as the canonical dimensions and the I.R. dimensions

\begin{tabular}{|r|r|r|r|r|r|}
\hline & $A_{\mu}$ & $B_{\mu \nu}$ & $b$ & $b_{\mu}$ & $e$ \\
\hline I.R.dim & 2 & 1 & 1 & 2 & 1 \\
\hline
\end{tabular}

It is also clear that the propagators in (73) are analytic in the $k$ parameter

\section{Appendix B}

We briefly consider the renormalization of the nilpotency condition

$$
\widehat{D} \Gamma^{(n)} \equiv \int d^{4} x\left(\partial_{\mu} \theta^{a} \frac{\delta}{\delta \widehat{\gamma}_{\mu}^{a}}+\partial_{\mu} A^{a \mu} \frac{\delta}{\delta \xi^{a}}\right) \Gamma^{(n)}=0
$$


via the Q.A.P at the first non-trivial order we have

$$
\widehat{D} \Gamma^{(n)}=X^{(n)}+O\left(\hbar^{(n+1)}\right)
$$

where $X^{(n)}$ is a local functional of canonical dimension $\leq 2$, carrying two units of $\Phi \Pi$ charge and respecting the constraints (20). Accordingly it can be parametrized as

$$
X^{(n)}=\int d^{4} x\left(L^{a[b c]} \partial_{\mu} A^{a \mu} \theta^{b} \theta^{c}+R^{a(b c)} A^{a \mu} \theta^{b} \partial_{\mu} \theta^{c}\right)
$$

and the square (round) brackets denote symmetrization (antisymmetrization). We easily find that

$$
X^{(n)}=\widehat{D} \widehat{X}^{(n)}
$$

with

$$
\widehat{X}^{(n)}=\int d^{4} x\left(L^{a[b c]} \xi^{a} \theta^{b} \theta^{c}+R^{a(b c)} A^{a \mu} \theta^{b} \widehat{\gamma}_{\mu}^{c}\right)
$$

which amounts to the compensability condition of the breaking $X^{(n)}$.

\section{Appendix $\mathrm{C}$}

In order to identify the local cohomology of the $\widehat{S}_{L}$ operator we filter with the counting operator [12]

$$
N=\int d^{4} x\left(A^{a \mu} \frac{\delta}{\delta A^{a \mu}}+\widehat{\gamma}^{a \mu \nu} \frac{\delta}{\delta \widehat{\gamma}^{a \mu \nu}}+\theta^{a} \frac{\delta}{\delta \theta^{a}}+\eta^{a} \frac{\delta}{\delta \eta^{a}}+\widehat{\Omega}^{a \mu} \frac{\delta}{\delta \widehat{\Omega}^{a \mu}}\right)
$$

and accordingly we have

$$
\widehat{S}_{L}=S^{(0)}+S^{(1)}+S^{(2)}+S^{(3)}
$$

which obey

$$
\begin{aligned}
\left(S^{(0)}\right)^{2} & =0 \\
\left\{S^{(0)}, S^{(1)}\right\} & =-k D
\end{aligned}
$$




$$
\begin{aligned}
\left\{S^{(0)}, S^{(2)}\right\}+\left(S^{(1)}\right)^{2} & =0 \\
\left\{S^{(0)}, S^{(3)}\right\}+\left\{S^{(1)}, S^{(2)}\right\} & =0 \\
\left(S^{(2)}\right)^{2}+\left\{S^{(1)}, S^{(3)}\right\} & =0 \\
\left\{S^{(2)}, S^{(3)}\right\} & =0 \\
\left(S^{(3)}\right)^{2} & =0
\end{aligned}
$$

and $S^{(0)}$ is explicitely given by

$$
\begin{aligned}
S^{(0)} & =\int d^{4} x\left(\partial_{\mu} \theta^{a} \frac{\delta}{\delta A^{a \mu}}+\varepsilon^{\mu \nu \rho \sigma} \partial_{\nu} B_{\rho \sigma} \frac{\delta}{\delta \widehat{\gamma}^{a \mu}}+2 \partial_{\mu} c^{a \nu} \frac{\delta}{\delta B^{a \mu \nu}}-\varepsilon^{\mu \nu \rho \sigma} \partial_{\rho} A_{\sigma}^{a} \frac{\delta}{\delta \widehat{\gamma}^{a \mu \nu}}\right. \\
& \left.-2 \partial^{\nu} \widehat{\gamma}_{\mu \nu}^{a} \frac{\delta}{\delta \widehat{\Omega}_{\mu}^{a}}+\partial_{\mu} \widehat{\gamma}^{a \mu} \frac{\delta}{\delta \xi^{a}}-\partial_{\mu} \widehat{\Omega}^{a \mu} \frac{\delta}{\delta \eta^{a}}+\partial_{\mu} d^{a} \frac{\delta}{\delta c_{\mu}^{a}}\right)
\end{aligned}
$$

When operating on functions, $S^{(0)}$ can be rewritten as an ordinary differential operator; setting

$$
\begin{aligned}
A_{\mu, \nu}^{+a} & =\frac{1}{2}\left(\partial_{\mu} A_{\nu}^{a}+\partial_{\nu} A_{\mu}^{a}\right) \\
A_{\mu, \nu}^{-a} & =\frac{1}{2}\left(\partial_{\mu} A_{\nu}^{a}-\partial_{\nu} A_{\mu}^{a}\right) \\
c_{\mu, \nu}^{+a} & =\frac{1}{2}\left(\partial_{\mu} c_{\nu}^{a}+\partial_{\nu} c_{\mu}^{a}\right) \\
c_{\mu, \nu}^{-a} & =\frac{1}{2}\left(\partial_{\mu} c_{\nu}^{a}-\partial_{\nu} c_{\mu}^{a}\right) \\
B_{\mu \nu, \rho}^{-a} & =\frac{1}{3}\left(\partial_{\rho} B_{\mu \nu}^{a}+\partial_{\mu} B_{\nu \rho}^{a}+\partial_{\nu} B_{\rho \mu}^{a}\right) \\
B_{\mu \nu, \rho}^{+a} & =\frac{1}{3}\left(2 \partial_{\rho} B_{\mu \nu}^{a}-\partial_{\mu} B_{\nu \rho}^{a}-\partial_{\nu} B_{\rho \mu}^{a}\right)
\end{aligned}
$$

and denoting the space-time partial derivatives of the fields with an index after a comma, we have

$$
\begin{aligned}
S^{(0)} & =\theta^{a, \mu} \frac{\partial}{\partial A^{a \mu}}+\theta^{a, \mu \nu} \frac{\partial}{\partial A^{+a \mu, \nu}}+\theta^{a, \mu \nu \rho} \frac{\partial}{\partial A^{+a \mu, \nu \rho}} \\
& +\varepsilon_{\mu \nu \rho \sigma}\left(B^{-a \nu \rho, \sigma} \frac{\partial}{\partial \widehat{\gamma}^{a \mu}}+B^{-a \nu \rho, \sigma \tau} \frac{\partial}{\partial \widehat{\gamma}^{a \mu, \tau}}\right) \\
& +4\left(c^{-a \mu, \nu} \frac{\partial}{\partial B^{a \mu \nu}}+c^{-a \mu, \nu \rho} \frac{\partial}{\partial B^{+a \mu \nu, \rho}}+c^{-a \mu, \nu \rho \sigma} \frac{\partial}{\partial B^{+a \mu \nu, \rho \sigma}}\right) \\
& -2 \varepsilon^{\mu \nu \rho \sigma}\left(A_{\rho, \sigma}^{-a} \frac{\partial}{\partial \widehat{\gamma}^{a \mu \nu}}+A_{\rho, \sigma \tau}^{-a} \frac{\partial}{\partial \widehat{\gamma}^{a \mu \nu, \tau}}+A_{\rho, \sigma \tau \lambda}^{-a} \frac{\partial}{\partial \widehat{\gamma}^{a \mu \nu, \tau \lambda}}\right)
\end{aligned}
$$




$$
\begin{aligned}
& +\widehat{\gamma}_{\mu}^{a \mu} \frac{\partial}{\partial \xi^{a}}-\widehat{\Omega}_{\mu}^{a \mu} \frac{\partial}{\partial \eta^{a}}+2\left(\widehat{\gamma}_{\nu}^{a \mu \nu} \frac{\partial}{\partial \widehat{\Omega}^{a \mu}}+\widehat{\gamma}_{\nu}^{a \mu \nu, \tau} \frac{\partial}{\partial \widehat{\Omega}^{a \mu, \tau}}\right) \\
& +\partial d^{a, \mu} \frac{\partial}{\partial c^{a \mu}}+d^{a, \mu \nu} \frac{\partial}{\partial c^{+a \mu, \nu}}+d^{a, \mu \nu \rho} \frac{\partial}{\partial c^{+a \mu, \nu \rho}}+d^{a, \mu \nu \rho \sigma} \frac{\partial}{\partial c^{+a \mu, \nu \rho \sigma}}
\end{aligned}
$$

In the above expression all indices after the comma are completely simmetrized and we have taken into account that $S^{(0)}$ acts on functions of canonical dimension $\leq 4$. In this space we can define $S^{(0) \dagger}$ as

$$
\begin{aligned}
S^{(0) \dagger} & =A^{a \mu} \frac{\partial}{\partial \theta^{a, \mu}}+A^{+a \mu, \nu} \frac{\partial}{\partial \theta^{a, \mu \nu}}+A^{+a \mu, \nu \rho} \frac{\partial}{\partial \theta^{a, \mu \nu \rho}} \\
& +\varepsilon_{\mu \nu \rho \sigma}\left(\widehat{\gamma}^{a \mu} \frac{\partial}{\partial B^{-a \nu \rho, \sigma}}+\widehat{\gamma}^{a \mu, \tau} \frac{\partial}{\partial B^{-a \nu \rho, \sigma \tau}}\right) \\
& +4\left(B^{a \mu \nu} \frac{\partial}{\partial c^{-a \mu, \nu}}+B^{+a \mu \nu, \rho} \frac{\partial}{\partial c^{-a \mu, \nu \rho}}+B^{+a \mu \nu, \rho \sigma} \frac{\partial}{\partial c^{-a \mu, \nu \rho \sigma}}\right) \\
& -2 \varepsilon_{\mu \nu \rho \sigma}\left(\widehat{\gamma}^{a \mu \nu} \frac{\partial}{\partial A_{\rho, \sigma}^{-a}}+\widehat{\gamma}^{a \mu \nu, \tau} \frac{\partial}{\partial A_{\rho, \sigma \tau}^{-a}}+\widehat{\gamma}^{a \mu \nu, \tau \lambda} \frac{\partial}{\partial A_{\rho, \sigma \tau \lambda}^{-a}}\right) \\
& +\xi^{a} \frac{\partial}{\partial \widehat{\gamma}_{\mu}^{a \mu}}-\eta^{a} \frac{\partial}{\partial \widehat{\Omega}_{\mu}^{a \mu}}+2\left(\widehat{\Omega}^{a \mu} \frac{\partial}{\partial \widehat{\gamma}_{\nu}^{a \mu \nu}}+\widehat{\Omega}^{a \mu, \tau} \frac{\partial}{\partial \widehat{\gamma}_{\nu}^{a \nu \nu, \tau}}\right) \\
& +c^{a \mu} \frac{\partial}{\partial d^{a, \mu}}+c^{+a \mu, \nu} \frac{\partial}{\partial d^{a, \mu \nu}}+c^{+a \mu, \nu \rho} \frac{\partial}{\partial d^{a, \mu \nu \rho}}+c^{+a \mu, \nu \rho \sigma} \frac{\partial}{\partial d^{a, \mu \nu \rho \sigma}}
\end{aligned}
$$

Now the local cohomology of $S^{(0)}$ is identified by $X^{c h}(x)$, solution of

$$
\left\{S^{(0)}, S^{(0) \dagger}\right\} X^{c h}(x)=0
$$

By direct computation $\left\{S^{(0)}, S^{(0) \dagger}\right\}$ is an operator which counts all fields except $\theta^{a}, d^{a}$ without space time derivatives; therefore we find

$$
X^{c h}(x) \equiv X^{c h}\left(\theta^{a}(x), d^{a}(x)\right)
$$

The above result must be further restricted by the condition

$$
N^{a} X^{c h}(x)=0
$$

since all other constraints are already satisfied; this yields

$$
X^{c h}(x) \equiv X^{c h}\left(\theta^{a}(x)\right)
$$


Let us now return to the local cohomology of $\widehat{S}_{L}$; setting

$$
X(x)=\sum_{n=1} X^{(n)}
$$

we obtain for $X^{(n)}$ the recurrence

$$
S^{(0)} X^{(n)}(x)+S^{(1)} X^{(n-1)}(x)+S^{(2)} X^{(n-2)}(x)+S^{(3)} X^{(n-3)}(x)=0
$$

The solution of (91) is easily found if we observe that

$$
\begin{aligned}
S^{(1)} X^{c h}(x) & =\frac{1}{2} f^{a b c} \theta^{b} \theta^{c} \frac{\partial}{\partial \theta^{a}} X^{c h}(x) \\
S^{(2)} X^{c h}(x) & =0 \\
S^{(3)} X^{c h}(x) & =0
\end{aligned}
$$

and take into account the relations (81). Let us analyze the first few terms; for $n=1$ we have

$$
S^{(0)} X^{(1)}(x)=0
$$

with solution

$$
X^{(1)}(x)=S^{(0)} \widehat{X}^{(1)}(x)+X^{c h(1)}(\theta)
$$

which substituted into the next $(n=2)$ relation gives

$$
S^{(0)}\left(X^{(2)}(x)-S^{(1)} \widehat{X}^{(1)}(x)\right)+S^{(1)} X^{c h(1)}(x)=0
$$

Now the last term on the l.h.s. of the above equation still belongs to the cohomology of $S^{(0)}$ and therefore it must separetely vanish, leading to the solution

$$
\begin{aligned}
X^{(2)}(x) & =S^{(0)} \widehat{X}^{(2)}(x)+S^{(1)} \widehat{X}^{(1)}(x)+X^{c h(2)}(\theta) \\
\frac{1}{2} f^{a b c} \theta^{b} \theta^{c} \frac{\partial}{\partial \theta^{a}} X^{c h(1)}(x) & =0
\end{aligned}
$$

The procedure iterates and we find

$$
\begin{aligned}
& S^{(0)}\left(X^{(n+3)}(x)-S^{(1)} \widehat{X}^{(n+2)}(x)-S^{(2)} \widehat{X}^{(n+1)}(x)-S^{(3)} \widehat{X}^{(n)}(x)\right)=0 \\
& S^{(1)} X^{c h(n+2)}(x)=0
\end{aligned}
$$


which is the result given in the text.

Furthermore the general local functional $\widehat{\Gamma}^{0}$ of dimensions $\leq 4$, even G-parity, $\Phi \Pi$ charge -1 and obeying

$$
\begin{aligned}
\widehat{D} \widehat{\Gamma}^{(0)} & =0 \\
G^{a} \widehat{\Gamma}^{(0)} & =0 \\
\widehat{\Gamma}^{0} & \neq \widehat{S}_{L} \widehat{\widehat{\Gamma}}^{0}
\end{aligned}
$$

can be parametrized as:

$$
\begin{aligned}
\widehat{\Gamma}^{(0)} & =\int d^{4} x\left(a_{1} \widehat{\Omega}^{a \mu} c_{\mu}^{a}+a_{2} \widehat{\Omega}^{a \mu} \partial_{\mu} d^{a}+a_{3} \varepsilon^{\mu \nu \rho \sigma} \widehat{\gamma}_{\mu \nu}^{a} B a_{\rho \sigma}\right. \\
& +a_{4} f^{a b c} \widehat{\gamma}_{\mu \nu}^{a} \widehat{\gamma}^{b \mu \nu} \theta^{c}+a_{5} \varepsilon^{\mu \nu \rho \sigma} \widehat{\gamma}_{\mu \nu}^{a} \partial_{\rho} A^{a \sigma}+a_{6} f^{a b c} \varepsilon^{\mu \nu \rho \sigma} \widehat{\gamma}_{\mu \nu}^{a} A_{\rho}^{b} A_{\sigma}^{c} \\
& \left.+a_{7} \xi^{a} \theta^{a}+a_{8} \widehat{\gamma}^{a \mu} A_{\mu}^{a}\right)
\end{aligned}
$$

and from (98) we find $a_{1}=a_{2}=a_{3}=a_{7}=a_{8}=0$. The remaining three parameters are not independent since we still have to consider $\widehat{\Gamma}^{0}$ modulo terms of the type $\widehat{S}_{L} \widehat{\hat{\Gamma}}^{0}$ which do not contribute to the counterterms. Now

$$
\widehat{\widehat{\Gamma}}^{0} \propto \int d^{4} x \widehat{\gamma}_{\mu \nu}^{a} \widehat{\gamma}^{a \mu \nu}
$$

and we finally obtain that there are only two nonvanishing parameters, for instance $a_{4}, a_{5}$.

\section{References}

[1] V. I. Ogievetsky and I. V. Polubarinov, Yad. Fiz. 4(1968)210

S. Deser, Phys.Rev178(1969)1931

K. Hayashi, Phys.Lett.B44(1973)497

J. Kalb and P. Ramond, Phys.Rev.D9(1974)2273

Y. Nambu, Phys.Reports23(1976)250 
[2] W.Siegel Phys. Lett. 93B (1980) 275

D. Z. Freedman, P. K. Townsend, Nucl.Phys.B177(1981)282

P.K.Townsend, Phys.Lett.B88(1979)97

L.Baulieu J.Thierry-Mieg Phys.Lett 144B(1983) 221

L.Baulieu J.Thierry-Mieg Nucl. Phys.B 228(1985) 259

F.S.Fradkin and A.A.Tseytlin, Ann.Phys.162(1985)31

S. P. de Alwis, M. T. Grisaru and L. Mezincescu, Phys.Lett.B190(1987)122

N.K.Nielsen, Nucl.Phys.B332(1990)391

[3] S. P. de Alwis, M. T. Grisaru and L. Mezincescu, Nucl.Phys.B303(1988)57

[4] T.E.Clark,C.H.Lee and S.T.Love, Nucl.Phys.B308(1988)379

[5] G. T. Horowitz, Comm.Math.Phys.125(1989)417

G. T. Horowitz and M. Srednicki,Comm.Math.Phys.130(1990)83

M. Blau and G. Thompson, Ann.Phys.(N.Y.)205(1991)130

M. Blau and G. Thompson, Phys.Lett.B255(1991)535

[6] E. Guadagnini, N. Maggiore and S. P. Sorella, Phys.Lett.B255(1991)65

[7] N. Maggiore and S. P. Sorella, Nucl.Phys.B377(1992)236

N.Maggiore and S.P.Sorella preprint UGVA-DPT 1992/04-761

[8] J. Thierry-Mieg and L. Baulieu, Nucl.Phys.B228(1983)259

A. H. Diaz, Phs.lett.B203(1988)408

J. Thierry-Mieg, Nucl.Phys.B335(1990)334

[9] A. Blasi, O. Piguet and S.P. Sorella, Nucl.Phys.B356(1991)154

[10] J. Manes, R. Stora and B. Zumino, Comm.Math.phys.102(1985)157

B. Zumino, Nucl.Phys.B253(1985)477

[11] Y.M.P Lam, Phys.Rev.D6(1972)2145, 2161

T.E. Clark and J.H. Lowenstein, Nucl.Phys.B113(1976)109 
[12] J.A. Dixon, Cohomology and Renormalization of gauge theories, Imperial College preprint-1977

G. Bandelloni, J.Math.Phys.28(1987)2775

[13] B.de Witt J.W. van Holten Phys.Lett.79B(1979) 389

[14] G.Bandelloni J.Math.Phys 32(1991)3491 